\title{
Photoabsorption spectra of small cationic xenon clusters from time-dependent density functional theory
}

\author{
Micael J. T. Oliveira, ${ }^{1,2,3, a)}$ Fernando Nogueira, ${ }^{1}$ Miguel A. L. Marques, ${ }^{2,3, b)}$ and \\ Angel Rubio ${ }^{4,5,3}$ \\ ${ }^{1}$ Center for Computational Physics, University of Coimbra, Rua Larga, 3004-516 Coimbra, Portugal \\ ${ }^{2}$ Laboratoire de Physique de la Matière Condensé et Nanostructures, Université Lyon I, CNRS, UMR 5586, \\ Domaine Scientifique de la Doua, F-69622 Villeurbanne Cedex, France \\ ${ }^{3}$ European Theoretical Spectroscopy Facility (ETSF) \\ ${ }^{4}$ Dpto. Física de Materiales, Nano-Bio Spectroscopy group and ETSF Scientific Development Centre, \\ Universidad del País Vasco, Centro de Física de Materiales CSIC-UPV/EHU-MPC and DIPC, Av. Tolosa 72 , \\ E-20018 San Sebastián, Spain \\ ${ }^{5}$ Fritz-Haber-Institut der Max-Planck-Gesellschaft, Faradayweg 4-6 D-14 195 Berlin-Dahlem, Germany
}

(Received 21 April 2009; accepted 28 October 2009; published online 1 December 2009)

\begin{abstract}
Upon ionization, rare-gas (like $\mathrm{Ar}$ and $\mathrm{Xe}$ ) clusters shift their absorption spectrum from the ultraviolet to the visible. This happens as bonding becomes much stronger due to the removal of an electron from a strongly antibonding orbital. In this article, we study the absorption spectrum of small cationic xenon clusters $\left(\mathrm{Xe}_{n}^{+}\right.$, with $\left.n=3, \ldots, 35\right)$ by means of time-dependent density functional theory. These calculations include relativistic effects through the use of relativistic $j$-dependent pseudopotentials in a two-spinor formulation of the Kohn-Sham equations. The peak positions in our calculated spectra are in fairly good agreement with experiment and confirm that absorption is mainly due to a charged linear core composed of 3, 4, or $5 \mathrm{Xe}$ atoms where the positive charge is localized. However, we find large deviations concerning the oscillator strengths, which can be partially explained by the unsatisfactory treatment of exchange in common density functionals. Furthermore, we find that adequate ground-state geometries are necessary for the correct prediction of the qualitative features of the spectra. (C) 2009 American Institute of Physics.
\end{abstract}

[doi:10.1063/1.3265767]

\section{INTRODUCTION}

Neutral rare-gas clusters are van der Waals bound and their first electronic transition is in the ultraviolet region. However, when one electron is removed from the system, the bonding becomes much stronger and the absorption shifts to the visible region. In 1991, Haberland et al. ${ }^{1}$ measured the absorption cross section for $\mathrm{Xe}_{n}^{+}, 3 \leq n \leq 30$. They found that for all these clusters three broad peaks are observed in the 1-4 eV range, except for $\mathrm{Xe}_{3}^{+}$, where only two peaks are present. From these results they concluded that there were two isomeric structures for the xenon clusters present in the experiment. Theoretical calculations suggested that all cationic xenon clusters geometries were similar: ${ }^{2,3}$ a charged linear core with almost neutral atoms arranged in rings perpendicular to the core axis. The rings are located in the middle of the core bonds and contain up to five atoms. Because most of the charge is localized around the linear core, the shape of the absorption cross section is mainly determined by the core of the cluster. Thus, the two isomeric structures found in experiment must correspond to structures with a different number of atoms in the charged core. These results were confirmed by further experimental evidence and by theoretical calculations of the photoabsorption of cationic xenon clusters. ${ }^{4-7}$

\footnotetext{
${ }^{a)}$ Electronic mail: micael@teor.fis.uc.pt.

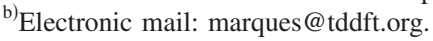

Up to now, most theoretical studies of cationic xenon clusters made use of semiempirical methods, especially the "diatomics in molecules" (DIM) approach. The DIM method $^{8}$ has been quite successful in describing Xe clusters, both for the ground state ${ }^{3,5}$ and their optical absorption. ${ }^{6,7}$ In this approach, a model Hamiltonian is built using high quality $a b$ initio diatomic data, such as energy surfaces of the neutral and the cationic dimer, both in the ground state and first excited state. Several extensions to the DIM model are required in order to accurately describe the electronic properties of cationic xenon clusters. These extensions include the spin-orbit coupling, three-body polarization contributions to the interaction energy, and three-body dispersion terms.

The scarcity of $a b$ initio calculations for these systems is probably due to several reasons. From a fundamental point of view, methods like density functional theory (DFT), with the standard approximations to the exchange-correlation functionals, are well capable of describing covalent bonding, but have problems with the long-range correlation ${ }^{9}$ present in these systems. From a more practical point of view, these are clusters with a large number of electrons where relativistic effects are of utmost importance. Indeed, inclusion of scalar relativistic effects is important to obtain correct ground state geometries, while a proper description of the absorption cross section also requires the inclusion of spin-orbit coupling. For example, if spin-orbit coupling is neglected, the $\mathrm{Xe}_{3}^{+}$absorption cross section only exhibits one peak. 
In fact, there are many interesting examples where relativistic effects are essential in the interaction of nanostructures with electromagnetic radiation. For instance, gold nanoclusters have been extensively studied as possible components for new optical devices ${ }^{10,11}$ and the importance of relativistic effects in gold is well known. ${ }^{12}$ The presence of heavy elements is not the only factor that makes relativistic effects important: spin-orbit coupling links the charge and spin dynamics of a system. This is very important for the field of spintronics, as it opens the possibility of controlling the spin of a system by means of electric fields. ${ }^{13}$ We should note, however, that most of what is known about the importance of relativistic effects in this kind of systems is mainly related to the ground state, while there are still many open questions regarding excited-state properties.

After the impressive success of DFT in describing the ground state properties of a wide variety of systems, timedependent DFT ${ }^{14,15}$ (TDDFT) is in turn becoming a widespread tool to investigate excited state properties of clusters and nanostructures. ${ }^{16}$ Like DFT, TDDFT offers a reasonable compromise between accuracy and computer efficiency, such that the theoretical study of complex phenomena in systems of several hundreds of atoms is now within our reach. Although the use of TDDFT to study excited states properties still poses several challenges, recent developments are extending the range of systems and phenomena where TDDFT yields accurate results. ${ }^{16}$

In this article, we present $a b$ initio calculations of the absorption spectrum of cationic Xe clusters using TDDFT. These calculations were made possible by the inclusion of spin-orbit in the code OCTOPUS ${ }^{17,18}$ using the recipe given in Ref. 19.

\section{TECHNICAL DETAILS}

All the calculations presented below are done considering the core electrons of xenon within a norm-conserving pseudopotential framework. The pseudopotentials were generated from the ground-state configuration $5 s^{2} 5 p^{6}$ of the free atom using the relativistic extension ${ }^{20}$ of the TroullierMartins method ${ }^{21}$ as implemented in the code APE. ${ }^{22}$ Spinorbit coupling was included using $j$-dependent pseudopotentials in the Kleinman and Bylander form. ${ }^{19,23}$ The cutoff radii used were $1.32,1.32$, and $1.69 \AA$ for the $s, p$, and $d$ components, respectively.

The optical absorption cross sections were computed using real-time propagation of the time-dependent Kohn-Sham equations $^{24}$ as implemented in the code OCTOPUs. ${ }^{17,18}$ The wave functions were represented on a real-space regular grid. A box composed of spheres of radius $4.5 \AA$ around every atom and a grid spacing of $0.42 \AA$ were required to obtain a convergence of better than $0.1 \mathrm{eV}$ in the spectra.

For linear $\mathrm{Xe}_{3}^{+}$and linear $\mathrm{Xe}_{4}^{+}$clusters we also optimized their geometries using DFT. We started our optimizations from previously published geometries ${ }^{3}$ and used the Broyden-Fletcher-Goldfarb-Shanno conjugate gradient algorithm with a convergence criterion of $10^{-3} \mathrm{eV} / \AA$ in the forces on every atom. The calculation of the forces with the necessary accuracy required the use of a grid spacing of 0.3
TABLE I. Calculated bond lengths $b$ angstrom of linear $\mathrm{Xe}_{3}^{+}$and linear $\mathrm{Xe}_{4}^{+}$. For the $\mathrm{Xe}_{4}^{+}$cluster $b_{2}$ denotes the central bond. The DIM results are from Ref. 3.

\begin{tabular}{lccc}
\hline \hline Cluster & Method & $b_{1}$ & $b_{2}$ \\
\hline $\mathrm{Xe}_{3}^{+}$ & LDA & 3.33 & \\
& PBE & 3.68 & \\
& DIM & 3.27 & \\
$\mathrm{Xe}_{4}^{+}$ & LDA & 3.47 & 3.43 \\
& PBE & 4.01 & 3.99 \\
& DIM & 3.55 & 3.18 \\
\hline \hline
\end{tabular}

$\AA$. To avoid unwanted effects from the boundaries during the optimization, a spherical box of radius $12 \AA$ was used. Due to reasons that will become apparent in the following, we used previously published geometries ${ }^{3}$ for all the other clusters.

\section{RESULTS AND DISCUSSION}

The linear $\mathrm{Xe}_{3}^{+}$and linear $\mathrm{Xe}_{4}^{+}$clusters are of particular interest, since they form the core of the larger clusters and previous studies suggested that the shape of the absorption cross section is mainly determined by this core. We thus started by optimizing the ground-state geometries of linear $\mathrm{Xe}_{3}^{+}$and linear $\mathrm{Xe}_{4}^{+}$using the Perdew-Wang parametrization of the local density approximation ${ }^{25}$ (LDA) and the generalized gradient approximation (GGA) of Perdew, Burke, and Ernzerhof $^{26}$ (PBE). Our results are summarized in Table I. We have also included for comparison in Table I the geometries obtained using the DIM semiempirical model, ${ }^{3}$ since to our knowledge no high quality ab initio result is available for these systems. Nevertheless, we expect the DIM geometries to be quite accurate, as a good agreement with experiment was obtained by DIM calculations of evaporation energies for $\mathrm{Xe}_{n}^{+} \rightarrow \mathrm{Xe}_{n-1}^{+}+\mathrm{Xe}$ (Ref. 3) and of the photoabsoption cross section of $\mathrm{Xe}_{n}^{+}$clusters. $^{7}$

These results indicate that, compared to the DIM geometries, the LDA overestimates considerably the bond lengths (with the exception of the $b_{1}$ bond in $\mathrm{Xe}_{4}^{+}$). As for the GGA, it produces larger bond lengths than the LDA. This is the typical behavior of the GGA, as the LDA usually underestimates the bond lengths, while the GGA overestimates them. Since in this case the LDA overestimate the bond lengths, it is not surprising that the GGA results appear to be worse. A possible reason for this overestimation is that both LDA and GGA fail to describe long-range correlation effects ${ }^{9}$ that should play an important role in the bonding of these systems and act to decrease the bond lengths.

Using the geometries from Table I we calculated the corresponding optical absorption spectra using TDDFT. For the LDA and PBE geometries the time propagation was carried using the same functional that was used to obtain the geometry. As for the geometries obtained with the DIM model, we computed the spectra using the LDA functional. The calculated spectra can be seen in Fig. 1. The overall structure of the spectra obtained with the LDA and DIM geometries is the same, although with differences in the po- 

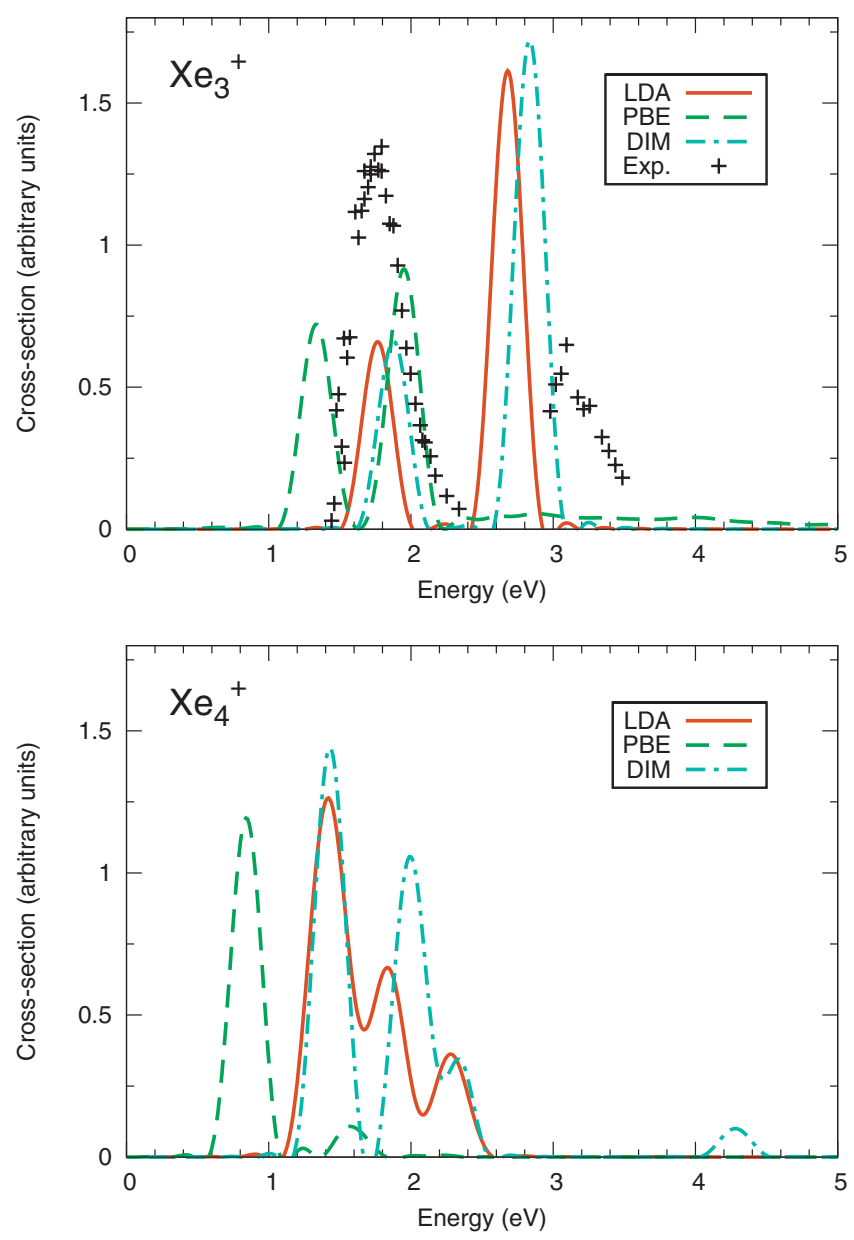

FIG. 1. Absorption cross section of linear $\mathrm{Xe}_{3}^{+}$(upper panel) and linear $\mathrm{Xe}_{4}^{+}$ (lower panel) calculated using LDA, PBE, and DIM geometries. The experimental results for $\mathrm{Xe}_{3}^{+}$from Ref. 1 are also plotted.

sition and intensities of the peaks. As for the spectra obtained with the PBE geometries, they present larger differences, specially in the case of linear $\mathrm{Xe}_{4}^{+}$.

We also included in Fig. 1 the experimental results for $\mathrm{Xe}_{3}^{+}$. The positions of the peaks obtained with LDA and DIM geometries are close to the experimental values and the error is close to the expected accuracy of the method $(0.1-0.4 \mathrm{eV})$. As for the PBE results, they seem to be worse than the LDA results. As it will be shown later, this is because of the differences in the geometries. We also note that the relative intensities of the two peaks appear to be inverted in all our calculated spectra ${ }^{1,7}$ and that the splitting due to spin-orbit coupling is underestimated; the experimental value is 1.35 $\mathrm{eV}$, but we obtain around $1.0 \mathrm{eV}$ with the LDA and DIM geometries, while the PBE one yields an even smaller value.

There are several possible sources of error in the relative intensities of the peaks of our calculated photoabsorption spectra. Two of them are the geometry and the exchange and correlation functional. Using a pseudopotential is in most cases a good approximation, but since the relative intensities are at least partly determined by the spin-orbit coupling, which is included through the pseudopotentials, it should also be considered as a possible source of error. Each of these possible sources of error was further investigated.

In order to check the effect of the geometry on the rela-

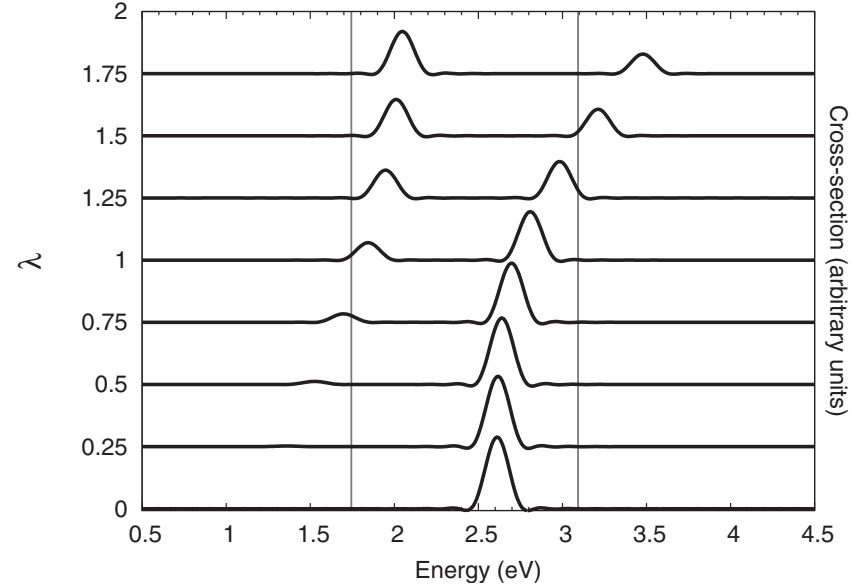

FIG. 2. Absorption cross section of linear $\mathrm{Xe}_{3}^{+}$for different values of the spin-orbit coupling strength $\lambda$ (see text for more details). The position of the two peaks of the experimental spectrum from Ref. 1 is indicated by gray vertical lines.

tive intensities of the peaks, we computed the photoabsorption spectra of $\mathrm{Xe}_{3}^{+}$for several bond lengths ranging from 3.1 to $3.9 \AA$. We verified that, in this range, the position of the peaks is much more sensitive to the geometry than the relative intensities. Indeed, only at large bond lengths $(3.9 \AA)$ did the relative intensities get inverted, but in that case the position of the peaks differs from experiment by more than $1 \mathrm{eV}$. This suggests that the error in the geometries is not the cause for the error in the relative intensities of the peaks.

Concerning the error arising from the pseudopotential, we first note that, in our calculations, all relativistic effects were included in the pseudopotential and that the valence electrons were subsequently treated using the nonrelativistic version of the (TD)DFT equations. This is justified by the fact that the Dirac equation for the valence states outside the core region reduces to the nonrelativistic Schrodinger equation. Nevertheless, important errors can be introduced if relativity is not properly taken into account during the construction of the pseudopotential. ${ }^{20}$ Although our pseudopotential was constructed using a scheme that should avoid this problem, we have performed the following tests to verify its quality. First, we computed the dipole matrix elements of the $\mathrm{Xe}^{+}$ valence states obtained from a fully relativistic all-electron calculation and compared them to the ones obtained from two pseudopotential calculations: one treating the valence states with the Schrödinger equation and the other one treating them fully relativistically using the Dirac equation. A very good agreement was found in all cases: the differences in the dipole-matrix elements obtained from the all-electron and pseudopotential calculations where smaller than $2.5 \%$, while the differences between the ones obtained from the two pseudopotential calculations where smaller than $0.01 \%$. Next, we computed the photoabsorption cross section of $\mathrm{Xe}_{3}^{+}$ using a modified Hamiltonian where the spin-orbit coupling term was multiplied by a prefactor $\lambda$. This term can be identified by writing the Kleinman and Bylander form for the $j$-dependent pseudopotentials in terms of the Pauli matrices, as shown in Appendix. In Fig. 2 we plot the optical absorption spectra of $\mathrm{Xe}_{3}^{+}$as a function of $\lambda$ considering a fixed 


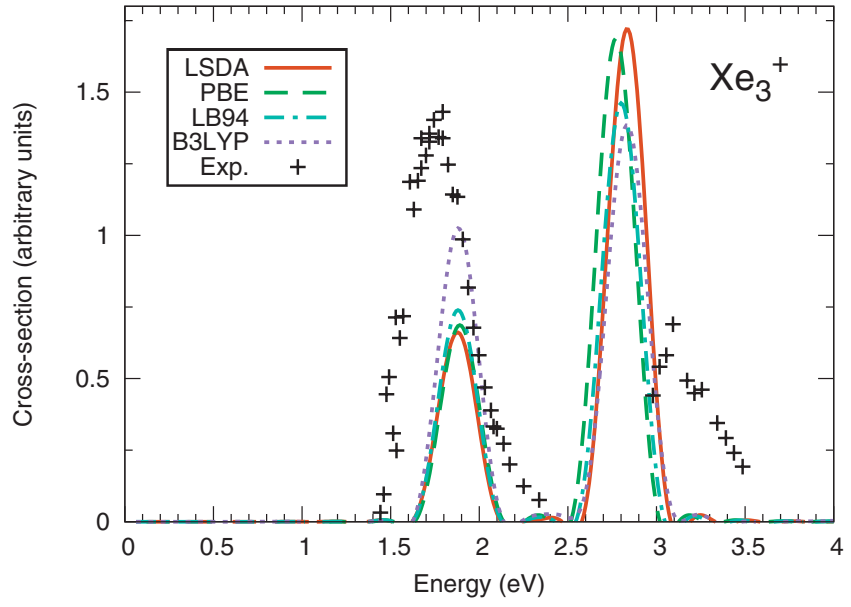

FIG. 3. Absorption cross section of linear $\mathrm{Xe}_{3}^{+}$calculated using the DIM geometry and different exchange-correlation functionals. The experimental results are from Ref. 1.

geometry. In this case the DIM geometry was used. As expected, for $\lambda=0$ there is only one peak present and a second peak at lower energy appears when $\lambda$ increases. From Fig. 2 we verify that, by varying $\lambda$, it is possible to modify and even invert the relative intensities of the peaks, but the best agreement between the calculated and measured values is obtained for a value of $\lambda$ between 1.5 and 1.75. Considering how well the pseudopotential is able to reproduce the fully relativistic all-electron results for $\mathrm{Xe}^{+}$, it seems unlikely that it could introduce an error such that it can justify the need to use such a large value of $\lambda$ to correct it.

Finally, in order to isolate the effect of the exchange and correlation functional in the spectra, we calculated the optical absorption spectra of $\mathrm{Xe}_{3}^{+}$using different exchange and correlation potentials, but keeping the geometries fixed. The geometries used were the ones obtained with the DIM model. Besides the LDA and PBE functional we also used the LB94 functional, ${ }^{27}$ which is a GGA with a correct asymptotic behavior, and the B3LYP hybrid functional. ${ }^{28}$ The results can be seen in Fig. 3. It turns out that, for all the functionals considered, the position of the peaks changes by less than $0.05 \mathrm{eV}$. Concerning the relative intensities of the peaks, the LDA and GGA functionals give very similar results, but B3LYP changes the relative intensities in the correct direction, although they are still inverted with respect to experiment. These results indicate that improving the treatment of the exchange part of the functional by adding a parcel of Hartree-Fock exchange reduces the error in the spectrum. One should note, however, that this is unrelated to the asymptotics of the exchange potential, as LB94 (that has the correct asymptotics) yields the same spectrum as the simple LDA and PBE. These results also confirm that the differences between LDA and GGA spectra in Fig. 1 come from the differences in the geometries. As a further analysis of the errors arising from the exchange and correlation functional, we computed the potential energy curve of the ground state of $\mathrm{Xe}_{2}^{+}$and performed a TDDFT calculation to obtain the energy differences with respect to the ground state of the two lowest dipole allowed transitions. We used the LDA and compared the curves thus obtained with the ones obtained by

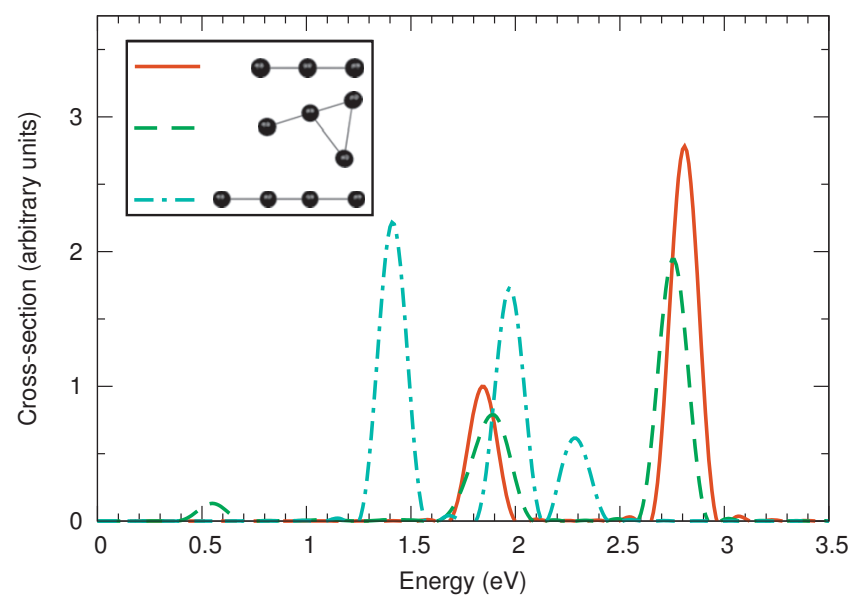

FIG. 4. Calculated photoabsorption cross section of linear $\mathrm{Xe}_{3}^{+}$, T-shaped $\mathrm{Xe}_{4}^{+}$, and linear $\mathrm{Xe}_{4}^{+}$within the TDLDA approximation using the geometries from Ref. 3 .

the coupled cluster calculations of Paidarová and Gadea. ${ }^{29}$ As expected, for large bond lengths the LDA curves are not able to reproduce the coupled cluster curves, but we find a very good agreement for bond lengths close to the equilibrium distance. Also, for all bond lengths, including the ones shorter than the equilibrium distance, the relative intensities of the transition dipole moments obtained with the LDA are inverted with respect to the coupled cluster results. This indicates that the error in the relative intensities is not related to the inadequate treatment of static correlation.

From the previous results, we conclude that adequate geometries are necessary in order to have a good agreement between the position of the peaks obtained from our calculations and the ones measured experimentally, while the error in the relative intensities of the peaks is most likely related to the approximate functional used in the TDDFT calculation. Because of this, and since our results seem to indicate that the spectra computed from the DIM geometries are more accurate than the spectra computed from DFT geometries, we have used the DIM geometries in all the remaining calculations. As for the functional used for the time propagations, although the results obtained with B3LYP are more accurate, we have used the LDA in the remaining calculations, as it gives the same excitation energies than B3LYP with a small modification of the oscillator strengths, and the usage of hybrid functionals in our real-space implementation of the TDDFT equations is computationally too expensive for systems with a large number of electrons.

In Fig. 4 we show the calculated spectra for $\mathrm{Xe}_{3}^{+}$and for two isomers of $\mathrm{Xe}_{4}^{+}$: a T-shaped geometry, which is the ground state, and a linear geometry. The spectra of $\mathrm{Xe}_{3}^{+}$and of the T-shaped $\mathrm{Xe}_{4}^{+}$, which has a trimer core, are very similar. On the other hand, the linear $\mathrm{Xe}_{4}^{+}$has a quite different structure with three distinct peaks that are redshifted with respect to $\mathrm{Xe}_{3}^{+}$and the T-shaped $\mathrm{Xe}_{4}^{+}$. This confirms previous experimental and theoretical work that showed that the absorption cross section of these clusters is mainly determined by the charged core.

More than one isomer is present in the experiment. ${ }^{1,4}$ This means that the existence of different metastable isomers 


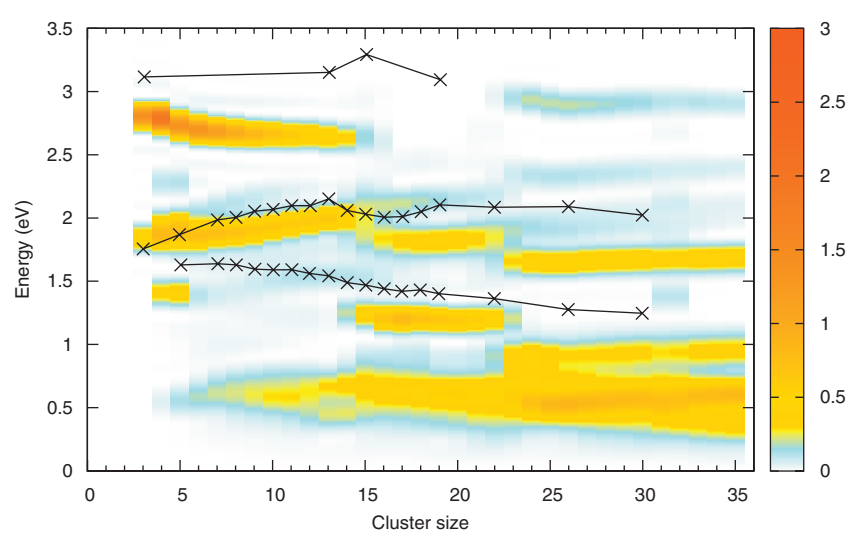

FIG. 5. Calculated photoabsorption cross section (arbitrary units) for $\mathrm{Xe}_{n}^{+}$, $3 \leq n \leq 35$, as a function of cluster size and energy. The positions of the peaks measured by Haberland et al. (Ref. 1) are indicated with crosses. The lines through the experimental data points are only to guide the eye.

needs to be taken into account in order to compare our results with experimental data. A crude way to simulate the absorption cross section at finite temperature is to sum the spectra of the different isomers using a Boltzmann distribution to weigh them. This, of course, neglects all effects in the spectra due to the vibration of the nuclei. For each cluster size, we have calculated the absorption cross section for all the local mimina found in the DIM calculations by Păska et $a{ }^{3} .^{3}$ and then used this method to obtain a spectra at finite temperature. In Fig. 5 we plot the absorption cross section, of $\mathrm{Xe}_{n}^{+}$as a function of $n$, for $3 \leq n \leq 35$. A temperature of 100 $\mathrm{K}$ was used for all cluster sizes, as the temperature in experiment was estimated between 60 and $100 \mathrm{~K}$. From the overall structure of the spectra, three regions can be clearly identified. These regions correspond to values of $n$ where different core sizes become more relevant in the spectra: trimer core for $3 \leq n \leq 13$, tetramer core for $14 \leq n \leq 22$, and pentamer core for $n \geq 23$.

We now analyze more in detail the trends in the positions of the main peaks. In our calculations, the position of the peak at around $3 \mathrm{eV}$ is systematically redshifted with respect to the experimental value. The reason for the disappearance of this peak for $n \geq 15$ is due to the fact that few isomers have been found in the DIM calculations for those clusters, ${ }^{3}$ as an extensive search in conformational space for such large clusters was prohibitive. The lack of experimental points for this peak makes it difficult to draw any further conclusions. As for the peak at around $2 \mathrm{eV}$, we see that for $n \leq 19$ our calculation is able to describe quite accurately the trend in the position of the peak. In particular, the change in slope at $n=13$ is well reproduced. The trend in the position of the lower energy peak measured in experiment is also reasonably well described, although the intensity of this peak is clearly underestimated. For all the peaks, at large values of $n$, comparison between our results and experiment becomes more difficult, as the structure of the spectra increases in complexity.

Finally, we note that in our calculation a broad peak can be found close to $0.5 \mathrm{eV}$. This peak is present for most isomers and becomes broader as the cluster size increases. This suggests that this low energy excitation is plasmonlike. Unfortunately there are no experimental measurements at these energies for us to compare with.

\section{CONCLUSIONS}

We studied the optical absorption of cationic xenon clusters using TDDFT, and showed that, provided that adequate ground-state geometries are used, TDDFT is able to correctly predict the qualitative features of the spectra. Our results are in agreement with previous theoretical calculations that concluded that the absorption cross section is mainly determined by the charged linear core of the clusters. Indeed, from our calculated spectra, the linear core of each isomer could clearly be identified. Even if the position of the peaks were well described, we found a poor agreement for the relative intensities of the peaks. The exchange and correlation functional was found to be responsible for this error.

\section{ACKNOWLEDGMENTS}

We thank R. Kalus and D. Hrivňák for providing us with the cationic xenon cluster geometries from Ref. 3 obtained using the DIM model. We thankfully acknowledge the computer resources, technical expertise, and assistance provided by the Laboratory for Advanced Computation of the University of Coimbra, by the Barcelona Supercomputing Center, and by GENCI (Project No. x2009096017). M.A.L. Marques acknowledges partial support by the Portuguese FCT through Project No. PTDC/FIS/73578/2006 and the French ANR (Grant No. ANR-08-CEXC8-008-01). M.J.T. Oliveira thankfully acknowledges financial support from the Portuguese FCT (Contract No. SFRH/BPD/44608/2008). A. Rubio acknowledges funding by the Spanish MEC (Grant No. FIS2007-65702-C02-01), "Grupos Consolidados UPV/EHU del Gobierno Vasco" (Grant No. IT-319-07), and the European Community through e-I3 ETSF project (Grant Agreement No. 211956).

\section{APPENDIX: KLEINMAN AND BYLANDER FORM FOR $j$-DEPENDENT PSEUDOPOTENTIALS}

The nonlocal part of the Kleinman and Bylander form for $j$-dependent pseudopotentials is given by ${ }^{19}$

$$
\hat{v}_{\mathrm{NL}}=\sum_{l, j, m_{j}} \frac{\left|\psi_{l, j, m_{j}}^{\mathrm{PP}} \delta v_{l, j}^{\mathrm{PP}}\right\rangle\left\langle\psi_{l, j, m_{j}}^{\mathrm{PP}} \delta v_{l j}^{\mathrm{PP}}\right|}{\left\langle\psi_{l, j, m_{j}}^{\mathrm{PP}}\left|\delta v_{l, j}^{\mathrm{PP}}\right| \psi_{l, j, m_{j}}^{\mathrm{PP}}\right\rangle}
$$

where $\left|\psi_{l, j, m_{j}}^{\mathrm{PP}}\right\rangle$ are the atomic pseudowave functions and $\delta v_{l, j}^{\mathrm{PP}}$ are the differences between the $j$-dependent pseudopotentials $v_{l, j}^{\mathrm{PP}}$ and an arbitrary local component. In Eq. (A1), the sum over $j$ runs from $j=l-\frac{1}{2}$ to $j=l+\frac{1}{2}$ while the sum over $m_{j}$ runs from $-j$ to $j$. The atomic pseudowave functions are in turn given by

$$
\left|\psi_{l, j, m_{j}}^{\mathrm{PP}}\right\rangle=\left|R_{l, j}^{\mathrm{PP}}\right\rangle\left|\Omega_{j, l, m_{j}}\right\rangle
$$

where $\left|R_{l, j}^{\mathrm{PP}}\right\rangle$ is the radial part of the pseudowave function and $\left|\Omega_{j, l, m_{j}}\right\rangle$ are the total angular momentum eigenfunctions. The $\left|\Omega_{j, l, m_{j}}\right\rangle$ can be written in terms of the spherical harmonics $\left|Y_{l}^{m}\right\rangle$ and the eigenfunctions of the $z$ component of the Pauli 
spin operator, $|\uparrow\rangle$ and $|\downarrow\rangle$. For $j=l+\frac{1}{2}, m_{j}=m+\frac{1}{2}$ they are given by

$$
\left|\Omega_{j, l, m_{j}}\right\rangle=\left(\frac{l+m+1}{2 l+1}\right)^{1 / 2}\left|Y_{l}^{m}\right\rangle|\uparrow\rangle+\left(\frac{l-m}{2 l+1}\right)^{1 / 2}\left|Y_{l}^{m+1}\right\rangle|\downarrow\rangle,
$$

while for $j=l-\frac{1}{2}, m_{j}=m-\frac{1}{2}$ we have

$$
\left|\Omega_{j, l, m_{j}}\right\rangle=\left(\frac{l-m+1}{2 l+1}\right)^{1 / 2}\left|Y_{l}^{m-1}\right\rangle|\uparrow\rangle-\left(\frac{l+m}{2 l+1}\right)^{1 / 2}\left|Y_{l}^{m}\right\rangle|\downarrow\rangle .
$$

In order to separate the spin-orbit part from the scalar relativistic one in Eq. (A1), we write the nonlocal part in terms of spherical harmonics and in terms of the Pauli matrices $\sigma_{x}, \sigma_{y}$, and $\sigma_{z}$. To do this we note that

$$
\begin{aligned}
& |\uparrow\rangle\langle\uparrow|=\frac{1}{2}\left(\sigma_{0}+\sigma_{z}\right), \\
& |\uparrow\rangle\langle\downarrow|=\frac{1}{2}\left(\sigma_{x}+\mathrm{i} \sigma_{y}\right), \\
& |\downarrow\rangle\langle\uparrow|=\frac{1}{2}\left(\sigma_{x}-\mathrm{i} \sigma_{y}\right), \\
& |\downarrow\rangle\langle\downarrow|=\frac{1}{2}\left(\sigma_{0}-\sigma_{z}\right),
\end{aligned}
$$

with $\sigma_{0}$ being the unity matrix. By making the appropriate changes in the summation ranges, and after some straightforward manipulations, we get

$$
\begin{aligned}
\hat{v}_{\mathrm{NL}}= & \sum_{l}\left\{\sum _ { m = - l } ^ { l } \left[\left(\frac{l+1}{2 l+1} \sigma_{0}+\frac{m}{2 l+1} \sigma_{z}\right) \hat{P}_{l, l+1 / 2}^{m, m}\right.\right. \\
& \left.+\left(\frac{l}{2 l+1} \sigma_{0}-\frac{m}{2 l+1} \sigma_{z}\right) \hat{P}_{l, l-1 / 2}^{m, m}\right] \\
& +\sum_{m=-l-1}^{l} \frac{\sqrt{(l+m+1)(l-m)}}{2(2 l+1)}\left[( \sigma _ { x } + \mathrm { i } \sigma _ { y } ) \left(\hat{P}_{l, l+1 / 2}^{m, m+1}\right.\right. \\
& \left.\left.\left.-\hat{P}_{l, l-1 / 2}^{m, m+1}\right)+\left(\sigma_{x}-\mathrm{i} \sigma_{y}\right)\left(\hat{P}_{l, l+1 / 2}^{m+1, m}-\hat{P}_{l, l-1 / 2}^{m+1, m}\right)\right]\right\},
\end{aligned}
$$

where

$$
\hat{P}_{l, j}^{m_{1}, m_{2}}=\frac{\left|R_{l, j}^{\mathrm{PP}} Y_{l}^{m_{1}} \delta v_{l, j}^{\mathrm{PP}}\right\rangle\left\langle R_{l, j}^{\mathrm{PP}} Y_{l}^{m_{2}} \delta v_{l, j}^{\mathrm{PP}}\right|}{\left\langle R_{l, j}^{\mathrm{PP}}\left|\delta v_{l, j}^{\mathrm{PP}}\right| R_{l, j}^{\mathrm{PP}}\right\rangle} .
$$

The spin-orbit coupling term in Eq. (A6) corresponds to the terms in $\sigma_{x}, \sigma_{y}$, and $\sigma_{z}$. Indeed, if we keep only the terms in $\sigma_{0}$ we end up with

$$
\hat{v}_{\mathrm{NL}}=\sum_{l} \sum_{m=-l}^{l}\left(\frac{l+1}{2 l+1} \hat{P}_{l, l+1 / 2}^{m, m}+\frac{l}{2 l+1} \hat{P}_{l, l-1 / 2}^{m, m}\right),
$$

which is just, as expected, the $j$-average of the $j$-dependent projectors.

${ }^{1}$ H. Haberland, B. von Issendorff, T. Kolar, H. Kornmeier, C. Ludewigt, and A. Risch, Phys. Rev. Lett. 67, 3290 (1991).

${ }^{2}$ M. Amarouche, G. Durand, and J. P. Malrieu, J. Chem. Phys. 88, 1010 (1988).

${ }^{3}$ P. Paška, D. Hrivňák, and R. Kalus, Chem. Phys. 286, 237 (2003).

${ }^{4}$ B. v. Issendorff, A. Hofmann, and H. Haberland, J. Chem. Phys. 111, 2513 (1999).

${ }^{5}$ J. A. Gascón, R. W. Hall, C. Ludewigt, and H. Haberland, J. Chem. Phys. 117, 8391 (2002).

${ }^{6}$ R. Kalus and D. Hrivňák, Chem. Phys. 278, 21 (2002).

${ }^{7}$ R. Kalus, D. Hrivňák, and P. Paška, Chem. Phys. 311, 287 (2005).

${ }^{8}$ F. O. Ellison, J. Am. Chem. Soc. 85, 3540 (1963).

${ }^{9}$ A. Marini, P. García-González, and A. Rubio, Phys. Rev. Lett. 96, 136404 (2006).

${ }^{10}$ S. Palomba, L. Novotny, and R. E. Palmera, Opt. Commun. 281, 480 (2008).

${ }^{11}$ J. Zheng, C. Zhang, and R. M. Dickson, Phys. Rev. Lett. 93, 077402 (2004).

${ }^{12}$ P. Pyykkö, Angew. Chem. Int. Ed. 43, 4412 (2004).

${ }^{13}$ S. Datta and B. Das, Appl. Phys. Lett. 56, 665 (1990).

${ }^{14}$ E. Runge and E. K. U. Gross, Phys. Rev. Lett. 52, 997 (1984).

${ }^{15}$ M. A. L. Marques and E. K. U. Gross, Annu. Rev. Phys. Chem. 55, 427 (2004).

${ }^{16}$ Time-Dependent Density Functional Theory, Lecture Notes in Physics, Vol. 706, edited by M. A. L. Marques, C. Ullrich, F. Nogueira, A. Rubio, K. Burke, and E. K. U. Gross (Springer, Berlin, 2006).

${ }^{17}$ M. A. L. Marques, A. Castro, G. F. Bertsch, and A. Rubio, Comput. Phys. Commun. 151, 60 (2003).

${ }^{18}$ A. Castro, H. Appel, M. Oliveira, C. A. Rozzi, X. Andrade, F. Lorenzen, M. A. L. Marques, E. K. U. Gross, and A. Rubio, Phys. Status Solidi B 243, 2465 (2006).

${ }^{19}$ G. Theurich and N. A. Hill, Phys. Rev. B 64, 073106 (2001).

${ }^{20}$ E. Engel, A. Hock, and S. Varga, Phys. Rev. B 63, 125121 (2001).

${ }^{21}$ N. Troullier and J. L. Martins, Phys. Rev. B 43, 1993 (1991).

${ }^{22}$ M. J. T. Oliveira and F. Nogueira, Comput. Phys. Commun. 178, 524 (2008).

${ }^{23}$ L. Kleinman and D. M. Bylander, Phys. Rev. Lett. 48, 1425 (1982).

${ }^{24}$ A. Castro, M. A. L. Marques, and A. Rubio, J. Chem. Phys. 121, 3425 (2004).

${ }^{25}$ J. P. Perdew and Y. Wang, Phys. Rev. B 45, 13244 (1992).

${ }^{26}$ J. P. Perdew, K. Burke, and M. Ernzerhof, Phys. Rev. Lett. 77, 3865 (1996).

${ }^{27}$ R. van Leeuwen and E. J. Baerends, Phys. Rev. A 49, 2421 (1994).

${ }^{28}$ P. J. Stephens, F. J. Devlin, C. F. Chabalowski, and M. J. Frisch, J. Phys. Chem. 98, 11623 (1994).

${ }^{29}$ I. Paidarová and F. X. Gadea, Chem. Phys. 274, 1 (2001). 